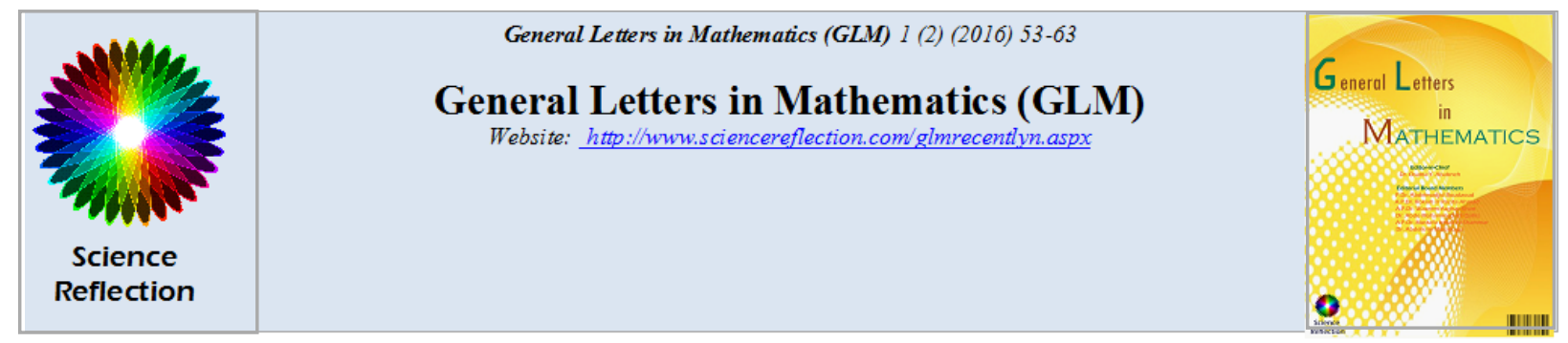

\title{
Some Types of $\mu$-semi-compactness Via hereditary classes
}

\author{
Abdo Qahis \\ Department of Mathematics, Faculty of Science and Arts \\ Nagran university, Saudi Arabia \\ E-mail: cahis82@gmail.com
}

\begin{abstract}
The aim of this paper is to introduce and study the notions of $\mu$-semicompactness and strong $\mu$-semi-compactness in generalized topological spaces with respect to a hereditary class, called $\mu \mathcal{H}$-semi-compactness and $\mathbf{S}-\mu \mathcal{H}$-semicompactness, respectively. Also several of their properties are presented. Finally some effects of various kinds of functions on them are studied..
\end{abstract}

Keywords: Generalized topology, hereditary class, $\mu$-semi-covering, $\mu \mathcal{H}$ semi-compact, $\mathbf{S}-\mu \mathcal{H}$-semi-compact.

2000 Mathematics Subject Classification. 54A05, 54A08, 54D10.

\section{Preliminaries and Introduction}

This work is developed around the concept of $\mu$-semi-compactness with respect a hereditary class which was introduced by Jamal M. Mustafa in [8]. In this research, we use the notions of generalized topology and hereditary class introduced by Császár in [1] and [2], respectively, in order to define and characterize the $\mu \mathcal{H}$-semi-compactness and $\mathbf{S}-\mu \mathcal{H}$-compactness spaces. Also some properties of these spaces are obtained and the behavior of these spaces under certain kinds of functions also is investigated. The strategy of using generalized topologies and hereditary classes to extend classical topological concepts have been used by many authors such as [2], [9], [11], [13].

Let $X$ be a non-empty set and $2^{X}$ denote the power set of $X$. We call a class $\mu \subseteq 2^{X}$ a generalized topology [1] (briefly, GT) if $\phi \in \mu$ and arbitrary union of elements of $\mu$ belongs to $\mu$. A set $X$ with a GT is called a generalized topological space (briefly, GTS) and is denoted by $(X, \mu)$. For a GTS $(X, \mu)$, the elements of $\mu$ are called $\mu$-open sets and the complement of $\mu$-open sets are called $\mu$-closed sets. For $A \subseteq X$, we denote by $c_{\mu}(A)$ the intersection of all $\mu$-closed sets containing $A$, i.e., the smallest $\mu$-closed set containing $A$ and 
by $i_{\mu}(A)$ the union of all $\mu$-open sets contained in $A$, i.e., the largest $\mu$-open set contained in $A$ (see [1], [3]). A subset $A$ of $X$ is said to be $\mu$-semi-compact if for every $\mu$-semi-open cover $\mathcal{U}$ of $A$ there exists a finite subfamily $\mathcal{U}^{\prime}$ that also covers $A$. $X$ is said to be $\mu$-semi-compact if $X$ is $\mu$-semi-compact as a subset[8].

A nonempty family $\mathcal{H}$ of subsets of $X$ is called a hereditary class [2] if $A \in \mathcal{H}$ and $B \subset A$ imply that $B \in \mathcal{H}$. Given a generalized topological space $(X, \mu)$ with a hereditary class $\mathcal{H}$, for a subset $A$ of $X$, the generalized local function of $A$ with respect to $\mathcal{H}$ and $\mu$ [2] is defined as follows: $A^{*}=\left\{x \in X: U \cap A \notin \mathcal{H}\right.$ for all $\left.U \in \mu_{x}\right\}$, where $\mu_{x}=\{U: x \in$ $U$ and $U \in \mu\}$. And for a subset $A$ of $X$, is defined: $c_{\mu}^{*}(A)=A \cup A^{*}$. The family $\mu^{*}=\left\{A \subset X: X \backslash A=c_{\mu}^{*}(X \backslash A)\right\}$ is a GT on $X$. The elements of $\mu^{*}$ are called $\mu^{*}$-open and the complement of a $\mu^{*}$-open set is called $\mu^{*}$-closed set. It is clear that a subset $A$ is $\mu^{*}$-closed if and only if $A^{*} \subset A$. If the hereditary class $\mathcal{H}$ satisfies the additional condition: if $A, B \in \mathcal{H}$ implies $A \cup B \in \mathcal{H}$, then $\mathcal{H}$ is called an ideal on $X[10]$. We call $(X, \mu, \mathcal{H})$ a hereditary generalized topological space and briefly we denote it by HGTS.

Definition 1.1 [3] Let $(X, \mu)$ generalized topological spaces. Then, $A \subseteq X$ is said to be $\mu$-semi-open set if and only if $A \subset c_{\mu} i_{\mu}(A)$ i.e., if there exist a $\mu$-open set $V \in \mu$, such that $V \subseteq A \subseteq c_{\mu}(V)$.

The complement of a $\mu$-semi-open set is said to be $\mu$-semi-closed. The collection of all $\mu$-semi-open set ( resp. $\mu$-semi-closed set ) sets in $X$ is denoted by $S O_{\mu}(X)\left(\right.$ resp : $\left.S C_{\mu}(X)\right)$. The $\mu$-semi-closure of $A$ in $(X, \mu)$ is the intersection of all $\mu$-semi-closed set containing $A$ and is denoted by $s c_{\mu}(A)$.

Definition 1.2 [11] Let $(X, \mu, \mathcal{H})$ hereditary generalized topological spaces and $A \subset X, A_{\mu}(\mathcal{H}, \mu)=\left\{x \in X: U \cap A \notin \mathcal{H} \quad\right.$ for $\quad$ every $\left.U \in S O_{\mu}(X, x)\right\}$ is called the generalized semi-local function of $A$ with respect to $\mathcal{H}$ and $\mu$, where $S O_{\mu}(X, x)=\left\{U \in S O_{\mu}(X): x \in U\right\}$.

We simply write $A_{\mu}$ instead of $A_{\mu}(\mathcal{H}, \mu)$ is this case there is no ambiguity. It is given in [14], $\mu^{* g}(\mathcal{H})$ is a generalized topology on $X$, generated by the subbases $\left\{U-H: U \in S O_{\mu}(X)\right.$ and $\left.H \in \mathcal{H}\right\}$ or equivalently $\mu^{* g}(\mathcal{H})=\{U \subset X:$ $\left.c_{\mu}^{* g}(X-U)=X-U\right\}$. The closure operator $c_{\mu}^{* g}$ for a generalized topology $\mu^{* g}(\mathcal{H})$ is defined as follows: for $A \subseteq X, c_{\mu}^{* g}(A)=A \cup A_{\mu} \cdot i_{\mu}^{* g}$ denotes the interior of the set $\mu^{* g}(\mathcal{H})$. It is know that $\mu \subseteq \mu^{*}(\mathcal{H}) \subseteq \mu^{* g}(\mathcal{H})$.

Definition 1.3 [14] A subset of $A$ of an hereditary generalized topological spaces $(X, \mu, \mathcal{H})$ is said to be:

1. $\mu$-semi-*-perfect if $A=A_{\mu}$;

2. $\mu$-semi dense in-itself if $A \subseteq A_{\mu}$; 
3. $\mu$-semi-*-closed if $A_{\mu} \subseteq A$.

Definition 1.4 Let $(X, \mu)$ be a GTS. Then a subset $A$ of $X$ is said to be $\mu$-dense [6] if $c_{\mu}(A)=X$. The space $(X, \mu)$ is said to be $\mu$-submaximal [7] if every $\mu$-dense subset is $\mu$-open in $X$.

Definition 1.5 [4] A GTS $(X, \mu)$ is said to be $\mu$-extremally disconnected if the $\mu$-closure of every $\mu$-open set is $\mu$-open

Definition 1.6 [1] Let $(X, \mu)$ and $(Y, \nu)$ be two GTS's, then a function $f:(X, \mu) \rightarrow(Y, \nu)$ is said to be $(\mu, \nu)$-continuous if $U \in \nu$ implies $f^{-1}(U) \in \mu$.

Definition 1.7 [8] Let $(X, \mu)$ and $(Y, \nu))$ be GTS's. A function $f:(X, \mu) \rightarrow$ $(Y, \nu)$ is said to be $\mu$-pre-semi-open if it maps $\mu$-semi-open subsets of $X$ onto $\mu$-semi-open subsets of $Y$.

\section{$2 \mu \mathcal{H}$-Semi-Compactness Spaces}

Definition 2.1 A subset $A$ of a $\operatorname{HGTS}(X, \mu, \mathcal{H})$ is said to be $\mu \mathcal{H}$-semicompact if for every $\mu$-semi-open cover $\left\{V_{\alpha}\right\}_{\alpha \in \Lambda}$ of $A$ there exists a finite subset $\Lambda_{0}$ of $\Lambda$ such that $A \backslash \bigcup_{\alpha \in \Lambda_{0}} V_{\alpha} \in \mathcal{H}$. The HGTS $(X, \mu, \mathcal{H})$ is said to be $\mu \mathcal{H}$ semi-compact if $X$ is $\mu \mathcal{H}$-semi-compact as a subset.

Remark 2.2 1. It is clear that $(X, \mu)$ is $\mu$-semi-compact if and only if $(X, \mu,\{\phi\})$ is $\mu\{\phi\}$-semi-compact.

2. If $(X, \mu)$ is a $\mu$-semi-compact then $(X, \mu, \mathcal{H})$ is $\mu \mathcal{H}$-semi-compact. The converse is not true as shown by the following example.

Example 2.3 Let $X=[0,1), \mu=\{\phi\} \cup\{[0, a): 0<a \leq 1\}$ and $\mathcal{H}=\{A$ : $A \subseteq[0,1)\}$, then:

1. $(X, \mu, \mathcal{H})$ is $\mu \mathcal{H}$-semi-compact because for any $\mu$-semi-open cover $\left\{V_{\alpha}\right\}_{\alpha \in \Lambda}$ we have $X \backslash \bigcup_{\alpha \in \Lambda} V_{\alpha} \in \mathcal{H}$;

2. $(X, \mu)$ is not $\mu$-semi-compact. In fact if we take $V_{n}=\left[0,1-\frac{1}{n}\right)$ for all integer number $n \geq 2$, then $X=\bigcup_{n \geq 2}\left[0,1-\frac{1}{n}\right)$ but if $k \in Z^{+}$where $Z^{+}$ is the set of positive integers, and $n_{1}, n_{2}, \ldots, n_{k}$ are integer numbers with $n_{i} \geq 2$, for all $i \in\{1,2, \ldots, k\}$, then $X \neq \bigcup_{i=1}^{k}\left[0,1-\frac{1}{n_{i}}\right)$.

It is clear that the family $S O_{\mu}(X)$ of all $\mu$-semi-open subsets of $X$ forms a generalized topology on $X$ which is finer than $\mu$. 
Proposition 2.4 Let $(X, \mu, \mathcal{H})$ be a HGTS and $\mathcal{B}$ be a base for the generalized topology $S O_{\mu}(X)$, then $(X, \mu, \mathcal{H})$ is $\mu \mathcal{H}$-semi-compact if and only if for all family $\left\{V_{\alpha}\right\}_{\alpha \in \Lambda}$ of $\mu$-semi-open sets in $\mathcal{B}$, if $X=\bigcup_{\alpha \in \Lambda} V_{\alpha}$ then there exists $\Lambda_{0} \subseteq \Lambda$, finite, with $X \backslash \bigcup_{\alpha \in \Lambda_{0}} V_{\alpha} \in \mathcal{H}$.

Proof. ( $\Longrightarrow)$ Let $\left\{V_{\alpha}\right\}_{\alpha \in \Lambda}$ be a family of non-empty $\mu$-semi-open subsets of $X$ such that $X=\bigcup_{\alpha \in \Lambda} V_{\alpha}$. Since $\mathcal{B}$ be a base for $S O_{\mu}(X)$, then $S O_{\mu}(X)=\{\cup B: B \subseteq \mathcal{B}\}$. So, for each $\alpha \in \Lambda$ there exists a family $\left\{B_{\alpha \beta}: \beta \in \Lambda_{\alpha}\right\} \subseteq \mathcal{B}$ such that $V_{\alpha}=\bigcup_{\beta \in \Lambda_{\alpha}} B_{\alpha \beta}$. Given that $X=\bigcup_{\alpha \in \Lambda} V_{\alpha}$ and $X=\bigcup_{\alpha \in \Lambda} \bigcup_{\beta \in \Lambda_{\alpha}} B_{\beta \alpha}$ is $\mu \mathcal{H}$-semi-compact there exist $B_{\alpha_{1} \beta_{1}}, B_{\alpha_{2} \beta_{2}}, \ldots, B_{\alpha_{k} \beta_{k}}$ such that $X \backslash \bigcup_{i=1}^{k} B_{\alpha_{i} \beta_{i}} \in \mathcal{H}$. But $X \backslash \bigcup_{i=1}^{k} V_{k} \subseteq X \backslash \bigcup_{i=1}^{k} B_{\alpha_{i} \beta_{i}}$ and so $X \backslash \bigcup_{i=1}^{k} V_{k} \in \mathcal{H}$. $(\Longleftarrow)$ It is obvious.

Given a generalized topological space $(X, \mu)$, we denote by $\mathcal{H}_{f}$ the hereditary class of finite subsets of $X$. The following proposition is obvious and thus the proof are omitted.

Proposition 2.5 For a $\operatorname{HGTS}(X, \mu, \mathcal{H})$ the following are equivalent:

1. $(X, \mu)$ is $\mu$-semi-compact;

2. $\left(X, \mu, \mathcal{H}_{f}\right)$ is $\mu \mathcal{H}_{f}$-semi-compact;

3. $(X, \mu,\{\phi\})$ is $\mu\{\phi\}$-semi-compact.

Theorem 2.6 A HGTS $(X, \mu, \mathcal{H})$ is $\mu \mathcal{H}$-semi-compact if and only if for any family $\left\{G_{\alpha}\right\}_{\alpha \in \Lambda}$ of $\mu$-semi-closed subsets of $X$ such that $\bigcap_{\alpha \in \Lambda} G_{\alpha}=\phi$, there exists a finite subset $\Lambda_{0}$ of $\Lambda$ with $\bigcap_{\alpha \in \Lambda_{0}} G_{\alpha} \in \mathcal{H}$.

Proof. Let $\left\{G_{\alpha}\right\}_{\alpha \in \Lambda}$ be a family of $\mu$-semi-closed subsets of $X$ such that $\bigcap_{\alpha \in \Lambda} G_{\alpha}=\phi$. Then $\left\{X \backslash G_{\alpha}\right\}_{\alpha \in \Lambda}$ is a $\mu$-semi-open cover of $X$. By hypothesis, there exists a finite subset $\Lambda_{0} \subset \Lambda$ such that $X \backslash \bigcup_{\alpha \in \Lambda_{0}}\left(X \backslash G_{\alpha}\right) \in \mathcal{H}$. This implies that $\bigcap_{\alpha \in \Lambda_{0}} G_{\alpha} \in \mathcal{H}$.

Conversely, Let $\left\{V_{\alpha}\right\}_{\alpha \in \Lambda}$ be any $\mu$-semi-pen cover of $X$. Then $\left\{X \backslash V_{\alpha}\right\}_{\alpha \in \Lambda}$ is a family of $\mu$-semi-closed subsets of $X$ such that $\bigcap_{\alpha \in \Lambda} X \backslash V_{\alpha}=\phi$. By hypothesis, there exists a finite subset $\Lambda_{0} \subset \Lambda$ such that $\bigcap_{\alpha \in \Lambda_{0}} X \backslash V_{\alpha} \in \mathcal{H}$. Consequently, we obtain that, $X \backslash \bigcup_{\alpha \in \Lambda_{0}} V_{\alpha} \in \mathcal{H}$ and therefore $(X, \mu, \mathcal{H})$ is $\mu \mathcal{H}$-semi-compact.

It is clear that the intersection of any two hereditary classes on a non-empty set $X$ is a heredity class. 
Corollary 2.7 If $\left(X, \mu, \mathcal{H}_{1} \cap \mathcal{H}_{2}\right)$ is $\mu\left(\mathcal{H}_{1} \cap \mathcal{H}_{2}\right)$-semi-compact, then $\left(X, \mu, \mathcal{H}_{1}\right)$ is $\mu \mathcal{H}_{1}$-semi-compact and $\left(X, \mu, \mathcal{H}_{2}\right)$ is $\mu \mathcal{H}_{2}$-semi-compact.

Definition 2.8 [5] A HGTS $(X, \mu, \mathcal{H})$ is said to be $\mu \mathcal{H}$-compact if for every $\mu$-open cover $\left\{V_{\alpha}\right\}_{\alpha \in \Lambda}$ of $X$ there exists a finite subset $\Lambda_{0}$ of $\Lambda$ such that $X \backslash$ $\bigcup_{\alpha \in \Lambda_{0}} V_{\alpha} \in \mathcal{H}$.

Proposition 2.9 Every $\mu \mathcal{H}$-semi-compact space is $\mu \mathcal{H}$-compact.

Proof. The proof is obvious since every $\mu$-open set is $\mu$-semi-open.

Proposition 2.10 If $(X, \mu)$ is $\mu$-submaximal and extremally disconnected, then $\mu \mathcal{H}$-compact and $\mu \mathcal{H}$-semi-compact are equivalent.

Proof. The proof comes immediately form the fact: In the extremally disconnected $\mu$-submaximal space $\mu=S O_{\mu}(X)$.

Next we study the behavior of some types of subspaces of a $\mu \mathcal{H}$-semicompact space relative to $X$.

Theorem 2.11 If $A_{i}, i=1,2$ are $\mu \mathcal{H}$-semi-compact subsets of a HGTS $(X, \mu, \mathcal{H})$, and $\mathcal{H}$ is an ideal then $A_{1} \cup A_{2}$ is $\mu \mathcal{H}$-semi-compact.

Proof. Let $\left\{V_{\alpha}\right\}_{\alpha \in \Lambda}$ be a family of $\mu$-semi-open subsets of $X$ such that $A_{1} \cup A_{2} \subseteq \bigcup_{\alpha \in \Lambda} V_{\alpha}$. Then $\left\{V_{\alpha}\right\}_{\alpha \in \Lambda}$ is a $\mu$-semi-open cover of $A_{i}$ for $i=1,2$. Since $A_{1}$ and $A_{2}$ are $\mu \mathcal{H}$-semi-compact, then there exists finite subsets $\Lambda_{0}$ and $\Lambda_{1}$ of $\Lambda$ with $A_{1} \backslash \bigcup_{\alpha \in \Lambda_{0}} V_{\alpha} \in \mathcal{H}$ and $A_{2} \backslash \bigcup_{\alpha \in \Lambda_{1}} V_{\alpha} \in \mathcal{H}$. This implies that $A_{1} \backslash \bigcup_{\alpha \in \Lambda_{0} \cup \Lambda_{1}} V_{\alpha} \in \mathcal{H}$ and $A_{2} \backslash \bigcup_{\alpha \in \Lambda_{0} \cup \Lambda_{1}} V_{\alpha} \in \mathcal{H}$ and since $\mathcal{H}$ is an ideal we have that

$\left(A_{1} \backslash \bigcup_{\alpha \in \Lambda_{0} \cup \Lambda_{1}} V_{\alpha}\right) \cup\left(A_{2} \backslash \bigcup_{\alpha \in \Lambda_{0} \cup \Lambda_{1}} V_{\alpha}\right) \in \mathcal{H}$. Thus $A_{1} \cup A_{2} \backslash \bigcup_{\alpha \in \Lambda_{0} \cup \Lambda_{1}} V_{\alpha} \in \mathcal{H}$. So $A_{1} \cup A_{2}$ is $\mu \mathcal{H}$-semi-compact.

The following example shows that the previous theorem does not hold when $\mathcal{H}$ is just a hereditary class, not an ideal.

Example 2.12 Let $X=(0,1)$, $\mu$ the usual topology, $\mathcal{H}=\{A \subset X: A \subset$ $\left(0, \frac{1}{2}\right)$ or $\left.A \subset\left(\frac{1}{2}, 1\right)\right\}$ and if $A=\left(0, \frac{1}{2}\right)$ and $B=\left(\frac{1}{2}, 1\right)$, then:

1. It is clear that $A=\left(0, \frac{1}{2}\right)$ and $B=\left(\frac{1}{2}, 1\right)$ are $\mu \mathcal{H}$-semi-compact subsets.

2. $A \cup B$ is not $\mu \mathcal{H}$-semi-compact if $\left\{\left(\frac{1}{n}, 1-\frac{1}{n}\right): n \in Z^{+}\right\}$is a cover of $\mu$-semi-open subsets of $X$ where $Z^{+}$is the set of positive integers, but if we choose a finite set $n_{1}, \ldots, n_{k}$ and we take $N=\max \left\{n_{1}, \ldots, n_{k}\right\}$, follows that $A \cup B \backslash \bigcup_{n=1}^{k}\left(\frac{1}{n_{i}}, \quad 1-\frac{1}{n_{i}}\right)=A \cup B \backslash\left(\frac{1}{N}, \quad 1-\frac{1}{N}\right) \notin \mathcal{H}$. 
A GTS $(X, \mu)$ is said to be $\mu$-semi-Hausdroff [12] for each pair of distinct points $x$ and $y$ in $X$, there exist $\mu$-semi-open sets $U_{x}$ and $V_{y}$ containing $x$ and $y$, respectively, such that $U \cap V=\phi$.

Lemma $2.13 x \notin A_{\mu}$ if and only if $(V \backslash H) \cap A=\phi$, where $V \in S O_{\mu}(X, x)$ and $H \in \mathcal{H}$.

Proof. Let $x \notin A_{\mu}$. Then there exist $U_{x} \in S O_{\mu}(X, x)$ such that $A \cap V=$ $H \in \mathcal{H}$. It follows that $(V \backslash H) \cap A=\phi$.

Conversely, suppose that $(V \backslash H) \cap A=\phi$ for some $V \in S O_{\mu}(X, x)$ and $H \in \mathcal{H}$. Then $V \cap(X \backslash H) \cap A=(A \cap V) \cap(X \backslash H)=\phi$. This implies that $(A \cap V) \subset H \in \mathcal{H}$. Hence, $x \notin A_{\mu}$.

Theorem 2.14 Let $(X, \mu, \mathcal{H})$ be a HGTS such that $(X, \mu)$ is a $\mu$-semiHausdorff. If $A$ is $\mu \mathcal{H}$-semi-compact subset of $X$, then $A$ is $\mu$-semi-*-closed.

Proof. Suppose that $A$ be a $\mu \mathcal{H}$-semi-compact subset of a $\mu$-semi-Hausdroff $\operatorname{HGTS}(X, \mu, \mathcal{H})$. Let $x \notin A$ then $x \in X \backslash A$. For each $y \in A$, there exist two $\mu$-semi-open sets $U_{y}$ and $V_{y}$ containing $x$ and $y$, respectively, such that $U_{y} \cap V_{y}=\phi$. Note that $x \notin s c_{\mu}\left(V_{y}\right)$. Then $\left\{V_{y}\right\}_{y \in A}$ is a $\mu$-semi-open cover of $A$ relative to $X$. Since $A$ is a $\mu \mathcal{H}$ - semi-compact, there exists a finite subset $\Lambda_{0}$ of $A$ such that $A \backslash \bigcup_{\Lambda_{0}} V_{y} \in \mathcal{H}$. Now $x \notin s c_{\mu}\left(V_{y}\right)$ for each $y \in A$ implies $x \notin \bigcup_{y \in \Lambda_{0}} s c_{\mu}\left(V_{y}\right)=s c_{\mu}\left(\bigcup_{y \in \Lambda_{0}} V_{y}\right)$. Let $U=X \backslash s c_{\mu}\left(\bigcup_{y \in \Lambda_{0}} V_{y}\right)$ and let $H=A \backslash s c_{\mu}\left(\bigcup_{y \in \Lambda_{0}} V_{y}\right) \subset A \backslash \bigcup_{y \in \Lambda_{0}} V_{y}=H_{1}$, where $H_{1} \in \mathcal{H}$. Since $U \in S O_{\mu}(X, x)$ and $H \in \mathcal{H}, U \backslash H \in \mu^{* g}(\mathcal{H})$ containing $x$ and $(U \backslash H) \cap A=\emptyset$. By Lemma 2.13, $x \notin A_{\mu}$. Hence $A_{\mu} \subset A$, so $A$ is $\mu$-semi-*-closed.

Let $(X, \mu, \mathcal{H})$ be a HGTS and let $A \subseteq X, A \neq \phi$. We denote by $\mathcal{H}_{A}$ the collection $\{H \cap A: H \in \mathcal{H}\}$ and by $\left(A, \mu_{A}\right)$ the subspace of $(X, \mu)$ on $A$. It is clear that the collection $\mu_{A}$ is a generalized topology on $A$ and the collection $\mathcal{H}_{A}$ is a hereditary class of subsets in $A$. Then we have the following theorem.

Theorem 2.15 Let $(X, \mu, \mathcal{H})$ be a $\mu \mathcal{H}$-semi-compact HGTS and $A$ be a $\mu$ semi-closed subset of $X$ then $\left(A, \mu_{A}, \mathcal{H}_{A}\right)$ is semi-compact with respect to the hereditary class $\mathcal{H}_{A}$.

Proof. Let $\left\{V_{\alpha}\right\}_{\alpha \in \Lambda}$ be a $\mu_{A}$-semi-open cover of $A$ where $V_{\alpha}=U_{\alpha} \cap A$ such that $U_{\alpha} \in S O_{\mu}(X)$. Then $\left\{U_{\alpha}\right\}_{\alpha \in \Lambda} \cup(X \backslash A)$ is a $\mu$-semi-covering of $X$ and hence there exists a finite subset $\Lambda_{0}$ of $\Lambda$ such that $X \backslash\left[\cup\left\{U_{\alpha}: \alpha \in \Lambda_{0}\right\} \cup(X \backslash\right.$ $A)] \in \mathcal{H}$. There exists $H \in \mathcal{H}$ with $X \backslash\left[\cup\left\{U_{\lambda}: \lambda \in \Lambda_{0}\right\} \cup(X \backslash A)\right]=H$. Since $A \cap H=A \cap\left(X \backslash\left[\cup\left\{U_{\alpha}: \alpha \in \Lambda_{0}\right\} \cup(X \backslash A)\right]\right)=A \cap\left(X \backslash \cup\left\{U_{\alpha}: \alpha \in \Lambda_{0}\right\}\right) \cap A=$ $A \cap\left(X \backslash \cup\left\{U_{\alpha}: \alpha \in \Lambda_{0}\right\}\right)=A \backslash \cup\left\{U_{\alpha}: \alpha \in \Lambda_{0}\right\}=A \backslash\left(A \cap\left[\cup\left\{U_{\alpha}: \alpha \in \Lambda_{0}\right\}\right]\right)=$ 
$A \backslash \cup\left\{U_{\alpha} \cap A: \alpha \in \Lambda_{0}\right\}$. Then, we have $A \backslash \cup\left\{U_{\alpha} \cap A: \alpha \in \Lambda_{0}\right\}=A \cap H \in \mathcal{H}_{A}$. This shows that $A$ is a $\mu \mathcal{H}_{\mathcal{A}^{-}}$semi-compact.

The well known result that a $\mu$-semi-closed subspace of a $\mu$-semi-compact space is $\mu$-semi-compact is a special case by taking $\mathcal{H}=\{\phi\}$.

Definition 2.16 Let $(X, \mu)$ be a GTS. Then a subset $A$ of $X$ is called a $\mu$ semi-generalized closed if $s c_{\mu}(A) \subseteq U$ whenever $A \subseteq U$ where $U$ is $\mu$-semi-open in $X$.

Theorem 2.17 If $(X, \mu, \mathcal{H})$ is $\mu \mathcal{H}$-semi-compact and $A \subset X$ is $\mu$-semi generalized-closed then $A$ is $\mu \mathcal{H}$-semi-compact.

Proof. Let $\left\{V_{\alpha}\right\}_{\alpha \in \Lambda}$ be any cover of $A$ by $\mu$-semi-open sets in $X$. Since $A$ is $\mu$-semi generalized-closed, $s c_{\mu}(A) \subset \cup V_{\alpha}$. Then $\left\{V_{\alpha}\right\}_{\alpha \in \Lambda} \cup\left(X \backslash s c_{\mu}(A)\right)$ is a $\mu$-semi-open cover of $X$ and hence there exists a finite subset $\Lambda_{0}$ of $\Lambda$ such that

$X \backslash\left[\bigcup_{\alpha \in \Lambda_{0}} V_{\alpha} \cup\left(X \backslash s c_{\mu}(A)\right] \in \mathcal{H}\right.$. Now, we have $X \backslash\left[\cup\left\{V_{\alpha}: \alpha \in \Lambda_{0}\right\} \cup(X \backslash\right.$ $\left.\left.s c_{\mu}(A)\right)\right]=\left[X \backslash \cup\left\{V_{\alpha}: \alpha \in \Lambda_{0}\right\}\right] \cap s c_{\mu}(A) \supset\left[X \backslash \cup\left\{V_{\alpha}: \alpha \in \Lambda_{0}\right\}\right] \cap A=$ $A \backslash\left[\cup\left\{V_{\alpha}: \alpha \in \Lambda_{0}\right\}\right]$. This implies that $A \backslash\left[\cup\left\{V_{\alpha}: \alpha \in \Lambda_{0}\right\}\right] \in \mathcal{H}$, and so $A$ is $\mu \mathcal{H}$-semi-compact relative to $X$.

Now we study the behavior of $\mu \mathcal{H}$-semi-compactness under certain types of functions.

Theorem 2.18 Let $(X, \mu)$ and $(Y, \nu)$ be GTSs and let $f:\left(X, S O_{\mu}(X)\right) \rightarrow$ $\left(Y, S O_{\nu}(Y)\right)$ be a $\left(S O_{\mu}(X), S O_{\nu}(Y)\right)$-continuous function. If $(X, \mu, \mathcal{H})$ is $\mu \mathcal{H}$ semi-compact, and if $\mathcal{G}=\left\{B \subseteq Y: f^{-1}(B) \in \mathcal{H}\right\}$ then:

1. $\mathcal{G}$ is a hereditary class on $Y$.

2. $(Y, \nu, \mathcal{G})$ is $\mu \mathcal{H}$-semi-compact.

Proof. (1) Suppose that $A \subseteq B \subseteq Y$ and $B \in \mathcal{G}$. Since $f^{-1}(A) \subseteq$ $f^{-1}(B) \in \mathcal{H}$, then $f^{-1}(A) \in \mathcal{H}$, and so $A \in \mathcal{G}$.

(2) Let $\left\{V_{\alpha}\right\}_{\alpha \in \Lambda} \subset S O_{\nu}(Y)$ such that $Y=\bigcup_{\alpha \in \Lambda} V_{\alpha}$. Since $X=\bigcup_{\alpha \in \Lambda} f^{-1}\left(V_{\alpha}\right)=$ $f^{-1}\left(\bigcup_{\alpha \in \Lambda} V_{\alpha}\right)$ and $(X, \mu, \mathcal{H})$ is $\mu \mathcal{H}$-semi-compact, there exists a finite subset $\Lambda_{0}$ of $\Lambda$ with $f^{-1}\left(Y \backslash \bigcup_{\alpha \in \Lambda_{0}} V_{\alpha}\right)=X \backslash \bigcup_{\alpha \in \Lambda_{0}} f^{-1}\left(V_{\alpha}\right) \in \mathcal{H}$. Thus $Y \backslash \bigcup_{\alpha \in \Lambda_{0}} V_{\alpha} \in \mathcal{G}$.

The following lemma is very useful in studying the preservation of $\mu \mathcal{H}$ compact by certain classes of functions 
Lemma 2.19 [5] Let $f:(X, \mu) \rightarrow(Y, \nu)$ be a function. If $\mathcal{H}$ is a hereditary class on $X$, then $f(\mathcal{H})=\{f(H): H \in \mathcal{H}\}$ is a hereditary class on $Y$.

Theorem 2.20 Let $(X, \mu)$ and $(Y, \nu)$ be GTSs and let $f:\left(X, S O_{\mu}(X)\right) \rightarrow$ $\left(Y, S O_{\nu}(Y)\right)$ be a surjection $\left(S O_{\mu}(X), S O_{\nu}(Y)\right.$-continuous function. If $(X, \mu, \mathcal{H})$ is $\mu \mathcal{H}$-semi-compact, then $(Y, \nu, f(\mathcal{H}))$ is $\nu f(\mathcal{H})$-semi-compact.

Proof. Let $\left\{V_{\alpha}\right\}_{\alpha \in \Lambda} \subset S O_{\nu}(Y)$ such that $Y=\bigcup_{\alpha \in \Lambda} V_{\alpha}$. Then $\left\{f^{-1}\left(V_{\alpha}\right)\right\}_{\alpha \in \Lambda}$ is a $\mu$-semi-open cover of $X$ and hence there exists a finite subset $\Lambda_{0}$ of $\Lambda$ such that $f^{-1}\left(Y \backslash \bigcup_{\alpha \in \Lambda_{0}} V_{\alpha}\right)=X \backslash \bigcup_{\alpha \in \Lambda_{0}} f^{-1}\left(V_{\alpha}\right) \in \mathcal{H}$. Thus $Y \backslash \bigcup_{\alpha \in \Lambda_{0}} V_{\alpha}=$ $f\left(f^{-1}\left(Y \backslash \bigcup_{\alpha \in \Lambda_{0}} V_{\alpha}\right)\right) \in f(\mathcal{H})$.

Corollary 2.21 Let $(X, \mu)$ and $(Y, \nu)$ be GTSs and let $f:\left(X, S O_{\mu}(X)\right) \rightarrow$ $\left(Y, S O_{\nu}(Y)\right)$ be a bijective $\mu$-semi-open function. If $(Y, \nu, \mathcal{G})$ is $\nu \mathcal{G}$-semi-compact, then $\left(X, \mu, f^{-1}(\mathcal{G})\right)$ is $\mu f^{-1}(\mathcal{G})$-semi-compact

Proof. Since $f:\left(X, S O_{\mu}(X)\right) \rightarrow\left(Y, S O_{\nu}(Y)\right)$ is a bijective $\mu$-semi-open function, $f^{-1}:\left(Y, S O_{\nu}(Y)\right) \rightarrow\left(X, S O_{\mu}(X)\right)$ is a surjection $\left(S O_{\mu}(X), S O_{\nu}(Y)\right)$ continuous function. Since $(Y, \nu, \mathcal{G})$ is $\nu \mathcal{G}$-semi-compact, by Theorem 2.20 we obtain that $\left(X, \mu, f^{-1}(\mathcal{G})\right)$ is $\mu f^{-1}(\mathcal{G})$-semi-compact.

\section{$3 \quad \mathrm{~S}-\mu \mathcal{H}$-Semi-Compactness Spaces}

Definition 3.1 If $(X, \mu, \mathcal{H})$ is a HGTS and $A \subseteq X, A$ is said to be strong $\mu \mathcal{H}$-semi-compact (briefly $\mathbf{S}$ - $\mu \mathcal{H}$-semi-compact) relative to $X$ if for every family $\left\{V_{\alpha}\right\}_{\alpha \in \Lambda}$ of $\mu$-semi-open subsets of $X$ with $A \backslash \bigcup_{\alpha \in \Lambda} V_{\alpha} \in \mathcal{H}$ there exists a finite subset $\Lambda_{0}$ of $\Lambda$, such that $A \subseteq \bigcup_{\alpha \in \Lambda_{0}} V_{\alpha}$. The $\operatorname{HGTS}(X, \mu, \mathcal{H})$ is said to be $\mathbf{S}-\mu \mathcal{H}$-semi-compact if $X$ is $\mathbf{S}-\mu \mathcal{H}$-semi-compact.

Clearly, the following diagram follows immediately from the definitions and facts.

$$
\begin{array}{cc} 
& \mu \mathcal{H}-\text { semi-compact } \\
\mathrm{S}-\mu \mathcal{H}-\text { semi-compact } & \uparrow \\
\searrow & \mu-\text { semi-compact }
\end{array}
$$

Remark 3.2 We note that if $(X, \mu, \mathcal{H})$ is a HGTS and $\left(X, \mu^{*}, \mathcal{H}\right)$ is a $\mathbf{S}$ $\mu \mathcal{H}$-semi-compact, then $(X, \mu, \mathcal{H})$ is $\mathbf{S}-\mu \mathcal{H}$-semi-compact, and that $(X, \mu, \mathcal{H})$ is $\mathbf{S}$ - $\mu \mathcal{H}$-semi-compact if and only if for any family $\left\{G_{\alpha}\right\}_{\alpha \in \Lambda}$ of $\mu$-semi-closed subsets of $X$, if $\bigcap_{\alpha \in \Lambda} G_{\alpha} \in \mathcal{H}$ then there exists $\Lambda_{0} \subseteq \Lambda$, finite, such that $\bigcap_{\alpha \in \Lambda_{0}} G_{\alpha}=\phi$. 
Remark 3.3 1. It is clear that the $G T(X, \mu)$ is $\mu$-semi-compact if and only if $(X, \mu,\{\phi\})$ is $\mathbf{S}-\mu\{\phi\}$-semi-compact.

2. If $(X, \mu, \mathcal{H})$ is a $\mathbf{S}$ - $\mu \mathcal{H}$-semi-compact then $(X, \mu, \mathcal{H})$ is $\mu \mathcal{H}$-semi-compact, and $(X, \mu)$ is $\mu$-semi-compact.

Definition 3.4 A subset $A$ of a $\operatorname{HGTS}(X, \mu, \mathcal{H})$ is said to be $S O_{\mu} \mathcal{H}_{g}$-closed if for every $U \in S O_{\mu}$ with $A \backslash U \in \mathcal{H}$ then $\operatorname{sc}_{\mu}(A) \subseteq U$.

Theorem 3.5 Every $S O_{\mu} \mathcal{H}_{g}$-closed subset of a $\mathbf{S}$ - $\mu \mathcal{H}$-semi-compact space is $\mathbf{S}-\mu \mathcal{H}$-semi-compact.

Proof. Let $A$ be any $S O_{\mu} \mathcal{H}_{g}$-closed subset of $(X, \mu, \mathcal{H})$ and $\left\{V_{\alpha}\right\}_{\alpha \in \Lambda}$ be a family of $\mu$-semi-open subsets of $X$ such that $A \backslash \bigcup_{\alpha \in \Lambda} V_{\alpha} \in \mathcal{H}$. Since $A$ is $S O_{\mu} \mathcal{H}_{g}$-closed, $s c_{\mu}(A) \subseteq \bigcup_{\alpha \in \Lambda} V_{\alpha}$. Then $\left(X \backslash s c_{\mu}(A)\right) \cup\left(\bigcup_{\alpha \in \Lambda} V_{\alpha}\right)$ is a $\mu$ semi-open cover of $X$ and so $X \backslash\left[X \backslash c_{\mu}(A) \cup\left(\bigcup_{\alpha \in \Lambda} V_{\alpha}\right)\right]=\phi \in \mathcal{H}$. Given that $X$ is $\mathbf{S}-\mu \mathcal{H}$-semi-compact there exists s finite subset $\Lambda_{0}$ of $\Lambda$ such that $X=\left(X \backslash s c_{\mu}(A) \cup \bigcup_{\alpha \in \Lambda_{0}} V_{\alpha}\right)$. Then $A=A \cap\left[\left(X \backslash s c_{\mu}(A)\right) \cup \bigcup_{\alpha \in \Lambda_{0}} V_{\alpha}\right]=$ $A \cap \bigcup_{\alpha \in \Lambda_{0}} V_{\alpha} \subseteq \bigcup_{\alpha \in \Lambda_{0}} V_{\alpha}$

Theorem 3.6 If $A$ and $B$ are $\mathbf{S}-\mu \mathcal{H}$-semi-compact subsets of a HGTS $(X, \mu, \mathcal{H})$, then $A \cup B$ is $\mathbf{S}-\mu \mathcal{H}$-semi-compact.

Proof. Let $\left\{V_{\alpha}\right\}_{\alpha \in \Lambda}$ be a family of $\mu$-semi-open subsets of $X$ such that $A \cup B \backslash \bigcup_{\alpha \in \Lambda} V_{\alpha} \in \mathcal{H}$. Since, $A \backslash \bigcup_{\alpha \in \Lambda} V_{\alpha} \subseteq A \cup B \backslash \bigcup_{\alpha \in \Lambda} V_{\alpha}$ and $B \backslash \bigcup_{\alpha \in \Lambda} V_{\alpha} \subseteq$ $A \cup B \backslash \bigcup_{\alpha \in \Lambda} V_{\alpha}$ then $A \backslash \bigcup_{\alpha \in \Lambda} V_{\alpha} \in \mathcal{H}$ and $B \backslash \bigcup_{\alpha \in \Lambda} V_{\alpha} \in \mathcal{H}$ and so there exist finite subsets $\Lambda_{0}$ and $\Lambda_{1}$ of $\Lambda$ such that $A \subseteq \bigcup_{\alpha \in \Lambda_{0}} V_{\alpha}$ and $B \subseteq \bigcup_{\alpha \in \Lambda_{1}} V_{\alpha}$. This implies that $A \subseteq \bigcup_{\alpha \in \Lambda_{0} \cup \Lambda_{1}} V_{\alpha}$ and $B \subseteq \bigcup_{\alpha \in \Lambda_{0} \cup \Lambda_{1}} V_{\alpha}$ and so $A \cup B \subseteq \bigcup_{\alpha \in \Lambda_{0} \cup \Lambda_{1}} V_{\alpha}$. Hence $A \cup B$ is $\mathbf{S}-\mu \mathcal{H}$-semi-compact.

Theorem 3.7 Let $(X, \mu, \mathcal{H})$ be a HGTS, $A \subseteq B \subseteq X$ and $B \subseteq s c_{\mu}(A)$. If $A$ is $\mu$-semi-generalized closed and $\mathbf{S}-\mu \mathcal{H}$-semi-compact, then $B$ is $\mathbf{S}-\mu \mathcal{H}$-semicompact.

Proof. Let $\left\{V_{\alpha}\right\}_{\alpha \in \Lambda}$ be a family of $\mu$-semi-open subsets of $X$ such that $B \backslash \bigcup_{\alpha \in \Lambda} V_{\alpha} \in \mathcal{H}$. Since, $A \backslash \bigcup_{\alpha \in \Lambda} V_{\alpha} \in \mathcal{H}$ and $A$ is $\mathbf{S}$ - $\mu \mathcal{H}$-semi-compact, there exists a finite subset $\Lambda_{0}$ of $\Lambda$ such that $A \subseteq \bigcup_{\alpha \in \Lambda_{0}} V_{\alpha}$. Since $A$ is $\mu$-semigeneralized closed, $s c_{\mu}(A) \subseteq \bigcup_{\alpha \in \Lambda_{0}} V_{\alpha}$ and this implies $B \subseteq \bigcup_{\alpha \in \Lambda_{0}} V_{\alpha}$.

Now we study the behavior of $\mathbf{S}-\mu \mathcal{H}$-semi-compactness under certain types of functions. 
Theorem 3.8 Let $(X, \mu)$ and $(Y, \nu)$ be GTSs and let $f:\left(X, S O_{\mu}(X)\right) \rightarrow$ $\left(Y, S O_{\nu}(Y)\right)$ be a $\left(S O_{\mu}(X), S O_{\nu}(Y)\right)$-continuous surjective function. If $(X, \mu, \mathcal{H})$ is $\mathbf{S}$ - $\mu \mathcal{H}$-semi-compact and $\mathcal{G}=\left\{B \subseteq Y: f^{-1}(B) \in \mathcal{H}\right\}$ then $(Y, \nu, \mathcal{G})$ is $\mathbf{S}$ $\mu \mathcal{H}$-semi-compact.

Proof. Let $\left\{V_{\alpha}\right\}_{\alpha \in \Lambda}$ be a family of $\mu$-semi-open subsets of $Y$ such that $Y \backslash \bigcup_{\alpha \in \Lambda} V_{\alpha} \in \mathcal{G}$. Since $X \backslash \bigcup_{\alpha \in \Lambda} f^{-1}\left(V_{\alpha}\right)=f^{-1}\left(Y \backslash \bigcup_{\alpha \in \Lambda} V_{\alpha}\right) \in \mathcal{H}$ and $(X, \mu, \mathcal{H})$ is $\mathbf{S}-\mu \mathcal{H}$-semi-compact, there exists a finite subset $\Lambda_{0}$ of $\Lambda$, such that $X=$ $\bigcup_{\alpha \in \Lambda_{0}} f^{-1}\left(V_{\alpha}\right)$. Given that $f$ is surjective we have $Y=\bigcup_{\alpha \in \Lambda_{0}} V_{\alpha}$.

Theorem 3.9 Let $(X, \mu)$ and $(Y, \nu)$ be GTSs and let $f:\left(X, S O_{\mu}(X)\right) \rightarrow$ $\left(Y, S O_{\nu}(Y)\right)$ be a bijective $\left(S O_{\mu}(X), S O_{\nu}(Y)\right)$-continuous function. If $(X, \mu, \mathcal{H})$ is $\mathbf{S}$ - $\mu \mathcal{H}$-semi-compact then $(Y, \nu, f(\mathcal{H}))$ is $\mathbf{S}-\nu f(\mathcal{H})$-semi-compact.

Proof. Let $\left\{V_{\alpha}\right\}_{\alpha \in \Lambda}$ be a family of $\mu$-semi-open subsets of $Y$ such that $Y \backslash \bigcup_{\alpha \in \Lambda} V_{\alpha} \in f(\mathcal{H})$. There exists $H \in \mathcal{H}$ with $Y \backslash \bigcup_{\alpha \in \Lambda} V_{\alpha}=f(H)$. Then $H=f^{-1}(f(H))=X \backslash \bigcup_{\alpha \in \Lambda} f^{-1}\left(V_{\alpha}\right) \in \mathcal{H}$. Given that $(X, \mu, \mathcal{H})$ is $\mathbf{S}-\mu \mathcal{H}$-semicompact, there exists a finite subset $\Lambda_{0}$ of $\Lambda$ such that $X=\bigcup_{\alpha \in \Lambda_{0}} f^{-1}\left(V_{\alpha}\right)$. Since $f$ is surjective, $Y=\bigcup_{\alpha \in \Lambda_{0}} V_{\alpha}$.

Corollary 3.10 If $f:(X, \mu) \rightarrow(Y, \nu)$ is a bijective and $\mu$-pre-semi-open function and $(Y, \nu, \mathcal{G})$ is $\mathbf{S}-\nu \mathcal{G}$-semi-compact, then $\left(X, \mu, f^{-1}(\mathcal{G})\right)$ is $\mathbf{S}-\mu f^{-1}(\mathcal{G})$ semi-compact.

Proof. Let $\left\{V_{\alpha}\right\}_{\alpha \in \Lambda}$ be a family of $\mu$-semi-open subsets of $X$ such that $X \backslash \bigcup_{\alpha \in \Lambda} V_{\alpha} \in f^{-1}(\mathcal{G})$. There exists $G \in \mathcal{G}$ with $X \backslash \bigcup_{\alpha \in \Lambda} V_{\alpha}=f^{-1}(G)$. Then $Y \backslash \bigcup_{\alpha \in \Lambda} f\left(V_{\alpha}\right)=f\left(f^{-1}(G)\right)$, and given that $(Y, \nu, \mathcal{G})$ is $\mathbf{S}$ - $\nu \mathcal{G}$-semi- compact then there exists a finite subset $\Lambda_{0}$ of $\Lambda$ with $Y=\bigcup_{\alpha \in \Lambda_{0}} f\left(V_{\alpha}\right)$. This implies that $X=\bigcup_{\alpha \in \Lambda_{0}} V_{\alpha}$.

ACKNOWLEDGEMENTS. The author would like to thank the referees for useful comments and suggestions.

\section{References}

[1] Császár Á., Generalized topology, generalized continuity, Acta Math. Hungar., 96 (2002), 351- 357. 
[2] Császár Á., Modification of generalized topologies via hereditary classes, Acta Math. Hungar., 115(1-2) (2007), 29 - 36.

[3] Császár Á. , Generalized open sets in generalized topologies, Acta Math. Hungar., 106(2005), 53-66.

[4] Császár Á., Extremally disconneted genealized topologies, Annal Univ. Sci. Budapest., 47 (2004), 91-96.

[5] Carpintero C., Rosas E., Salas-Brown M and Sanabria J., $\mu$-Compactness with respect to a hereditary class, Bol. Soc. Paran. Mat. 34(2) (2016), 231-236.

[6] Ekici E., Generalized hyperconnectedness, Acta Math. Hungar., 133(2011), 140-147.

[7] Ekici E. , Generalized submaximal spaces, Acta Math. Hungar., 134 (2012), 132-138.

[8] Jamal M. Mustafa, $\mu$-semi Compactness and $\mu$-semi Lindelofness in Generalized Topological Spaces, International journal of pure and applied mathematics, 78(4) (2012), 535 - 541.

[9] Kim Y. K. and Min W. K., On operations induced by hereditary classes on generalized topological spaces, Acta Math. Hungar., 137(1-2) (2012), $130-138$.

[10] Kuratowski K., Topologies I, Warszawa, (1933).

[11] Rajamani M., Inthumathi V. and Ramesh R.,Some new generalized topologies via hereditary classes, Bol. Soc. Paran. Mat., 30(2) (2012), 7177.

[12] Sarsak M.S., Weak separation axioms in generalized topological spaces, Acta Math. Hungar., 131(2011), 110-121.

[13] Zahram A. M., El-Saady K. and Ghareeb A.,Modification of weak structures via hereditary classes, Appl. Math. Letters., 25 (2012), 869-872.

[14] Hatir E., Noiri T.,On semi-I-I-open sets and semi-I-contnuous functions, Acta Math. Hungar., 2005, 107(4): 345-353. 